\title{
Actitudes de los escolares ante la llegada de población migrante: Estudio comparativo y aproximación al desarrollo de buenas prácticas educativas
}

\section{Attitudes of schoolchildren regarding the arrival of migrant population: Comparing and Prefiguring Proper Educational Practices}

\author{
Mohamed Chamsedine Habib Allah ${ }^{1 *}$ \\ mohamed.c.h@um.es, http://orcid.org/0000-0001-9817-652X \\ Azucena Hernández Martín** \\ azuher@usal.es, https://orcid.org/0000-0002-6731-7710 \\ * Universidad de Murcia, España \\ **Universidad de Salamanca, España
}

\section{Resumen:}

Algunos estudios señalan que los grupos sociales más próximos o en contacto con población migrante pueden desarrollar prejuicios, tanto explícitos como más sutiles que los que se generan entre poblaciones en las que el contacto con miembros de otras culturas es todavía poco significativo (Retortillo y Rodríguez, 2008; Cea D'Ancona, 2009; Checa y Arjona, 2011). Este trabajo tiene como objetivos conocer qué opinan y cuáles son las actitudes de los escolares ante la llegada de migrantes a las aulas y a otros contextos cercanos. Se han comparado dichas opiniones y actitudes en dos zonas españolas con distinta trayectoria en la presencia de migrantes, Salamanca y Murcia; analizando las posibles diferencias en dichas actitudes, atendiendo también al sexo y el nivel edu-

\begin{abstract}
:
Some studies point out that the social groups closer to or in contact with migrant populations may develop prejudices, both explicit and subtler than those generated between populations in which the contact with members of other cultures is still insignificant (Retortillo y Rodríguez, 2008; Cea D'Ancona, 2009; Checa y Arjona, 2011). The aim of this work is to find out what schoolchildren think about and what their attitudes are towards the arrival of migrant people to the classroom and other nearby settings. These opinions and attitudes have been compared in two Spanish areas with different trajectories in the presence of migrant people, Salamanca and Murcia. The possible differences in these attitudes were examined, also taking into account the gender and the educational
\end{abstract}

1 Dirección para correspondencia (correspondence address):

Mohamed Chamseddine Habib Allah. Facultad de Educación. Universidad de Murcia. Campus de Espinardo, s/n. 30100 Murcia (España). 
Actitudes de los escolares ante la llegada de población migrante: Estudio comparativo y aproximación al desarrollo de buenas prácticas educativas

Mohamed Chamseddine Habib Allah y Azucena Hernández Martín

cativo. La metodología incluye un estudio de carácter comparativo con una primera aproximación descriptiva, mediante la aplicación de un cuestionario. Los resultados indican la percepción generalizada del trato inadecuado que recibe el colectivo migrante, siendo las escolares quienes lo perciben en mayor medida. Con ciertos matices, la zona geográfica no es una variable que tenga importancia a la hora de considerar la influencia en clase de migrantes, como tampoco lo es el nivel educativo. Aunque las actitudes positivas son más frecuentes entre los escolares de Educación Primaria. El estudio finaliza apuntando algunas líneas de actuación educativa que fomenten la interacción y la convivencia positiva en el centro escolar.

\section{Palabras clave:}

Migración; intercambio cultural; escuela; formación; profesor. level of the participants. The methodology includes a comparative study with a first descriptive approximation, via a questionnaire. The results indicate the widespread perception, especially on the part of school children, that migrant groups are inappropriately treated. With certain nuances, geographic location and educational level are not important variables when it comes to considering the influence of migrant people on the class. However, positive attitudes are more frequent among Elementary Education students. The study concludes by highlighting educational actions that promote interaction and positive coexistence in schools.

\section{Key words:}

Migration; cultural exchange; school center; education; teacher.

\title{
Résumé:
}

Certaines études indiquent que les groupes sociaux les plus proches ou qui sont en contact avec une population migrante peuvent développer des préjuges, aussi bien explicites que plus subtiles que ceux qui sont générés dans les populations dans lesquelles le contact avec des membres d'autres cultures est encore peu significatif (Retortillo y Rodríguez, 2008; Cea D'Ancona, 2009; Checa y Arjona, 2011). Ce travail a pour objectif de connaître le point de vue des écoliers sur l'arrivée de migrants dans les classes et dans d'autres contextes proches. Ces opinions et attitudes ont été comparées dans deux zones espagnoles avec une trajectoire différentes en ce qui concerne la présence de collectifs migrants, Salamanque et Murcie, en analysant comparativement les éventuelles différences, en faisant attention aussi au sexe et au niveau éducatif. La méthodologie comprend une étude de caractère comparatif avec une première approche descriptive, au moyen de I'application d'un questionnaire. Les résultats indiquent que le traitement inadéquat généralement perçu que reçoit le collectif migrant, est ressenti de manière plus intense par les élèves de sexe féminin. Avec certaines nuances, la zone géographique et le niveau éducatif ne sont pas des variables qui ont une grande importance lorsqu'il s'agit de considérer leur influence sur une classe de migrants. Bien que les attitudes positives soient plus fréquentes entre les écoliers de l'enseignement primaire. L'étude indique quelques lignes d'action éducative qui contribuent à promouvoir l'interaction et la cohabitation positive dans le centre scolaire.

Mots clés: Migration; échange culturel; école; formation; professeur.

\author{
Fecha de recepción: 6-12-2017 \\ Fecha de aceptación: 17-9-2018
}




\section{Introducción}

En las tres últimas décadas asistimos en España a un aumento cuantitativo de los flujos de extranjeros que alcanza 4.549 .859 personas en los datos provisionales a 1 de enero de 2017, según el Instituto Nacional de Estadística. Sin embargo, la población extranjera no está distribuida de forma homogénea en las distintas Comunidades Autónomas, debido principalmente a razones vinculadas a las ofertas de empleo que ofrece cada zona geográfica.

En este contexto, se centra el interés en la Región de Murcia y en la Provincia de Salamanca, ya que son el objeto del estudio comparativo que se presentará en este trabajo. La primera cuenta con 1.469.656 habitantes donde se contabilizaba a fecha 1 de enero de 2017 un total de 197.737 habitantes extranjeros, de muy diversas procedencias. La segunda alcanza un total de 335.985 habitantes, de los cuales 11.656 son extranjeros procedentes de Latinoamérica y de África del norte, África subsahariana, sin olvidar la presencia de ciudadanos europeos ${ }^{2}$.

Dicha diferencia en cuanto a la presencia de población migrante en ambas localidades se refleja del mismo modo en los contextos escolares, teniendo en cuenta que la incorporación del alumnado migrante en las aulas de la Región de Murcia alcanza tres décadas. Sin embargo, en la provincia de Salamanca no supera una. En efecto, la Región de Murcia asciende a 35.717 alumnos y alumnas migrantes, y en Salamanca no sobrepasa los 2.150 estudiantes extranjeros, en el curso 2015/2016 (MECD, 2016).

En este sentido, la llegada de alumnado migrante está produciendo un mayor reto a la comunidad educativa para la gestión de la diversidad cultural en sus aulas. El profesorado la percibe como una dimensión particular, en ocasiones marginal y excluida de las prácticas de enseñanza, al considerar que se trata de un asunto de adaptación y una dificultad añadida que debe ser resuelta por el propio alumnado, con el apoyo de los orientadores y otros especialistas educativos (Carrasco y Coronel, 2017). Por otra parte, Pérez Serrano y Pérez de Guzmán (2011) advierten que la realidad social y educativa confirma que las diferencias humanas producen actitudes negativas, tensiones y prejuicios que se convierten

2 Datos obtenidos de la página del Instituto Nacional de Estadística. http://www.ine.es/ dyngs/INEbase/es/operacion.htm?c=Estadistica_C\&cid=1254736177000\&menu=res ultados\&idp $=1254735573002$ 
en rechazo, amenazas e incluso procesos violentos e irracionales y ponen en peligro la convivencia y la cohesión social en la escuela.

La multiculturalidad es un hecho pero la interculturalidad posee aún un modesto recorrido porque sigue siendo un desafío y un objetivo acuciante a conseguir. Desde esta perspectiva cabe señalar que sólo a partir del análisis e investigación ciertas actitudes es posible elaborar y acercarse a propuestas eficaces de prevención e intervención que permitan eliminar o disminuir las frecuentes predisposiciones negativas hacia estos colectivos que se reproducen en la escuela, como reflejo de otros contextos sociales. Estas medidas han de ser llevadas a cabo, tanto desde la administración educativa, como desde ámbitos educativos formales y no formales. El objetivo primordial es conseguir una actitud positiva hacia las personas migrantes, así como una mayor integración educativa y social de las mismas que posibilite un enriquecimiento intercultural.

Este estudio tiene, entre otros objetivos, conocer qué opinan los escolares ante la llegada de migrantes a las aulas y a otros contextos cercanos, en dos zonas geográficas españolas con distinta trayectoria en lo que respecta a la presencia de colectivos migrantes. Se parte de la idea de que aquellos grupos que se encuentran más en contacto con esta población pueden desarrollar prejuicios, ya no solamente explícitos, sino más sutiles que los que se generan entre poblaciones en las que el contacto con miembros de otras culturas es todavía poco significativo (Retortillo y Rodríguez, 2008).

\section{Marco teórico}

Como es bien sabido, la diversidad cultural es un hecho palpable en las sociedades europeas, produciéndose avances y retrocesos en relación a las interacciones que surgen cuando las personas de diferentes culturas comparten el mismo espacio y recursos, y teniendo en cuenta la gran diversidad de posiciones, intereses, necesidades y prioridades de los mismos. Es decir, el término multiculturalidad se refiere al conjunto, debidamente articulado, de elementos relacionados con la forma de pensar, sentir y actuar, ligado a creencias básicas y generales que dan a los diversos grupos culturales un grado más o menos elevado de cohesión. Este concepto no implica un enriquecimiento entre culturas. Sin embargo, la interculturalidad exige la comunicación comprensiva 
entre las diversas culturas que conviven en un mismo espacio, dándose un reconocimiento y valoración entre ellas, en un marco de igualdad de condiciones (De Juan, Parra y Beltrán, 2014).

Pero para ello, el contexto escolar debería atender la diversidad cultural como una riqueza y no un problema añadido para la comunidad educativa. En este sentido cabe resaltar la responsabilidad del pensamiento pedagógico y de la práctica educativa como ejercicio relacional y comunicación intercultural, para garantizar la inclusión educativa a este "nuevo" alumnado (Santos y Lorenzo, 2012).

Aunque esta situación de sensibilidad social y pedagógica sería lo deseable, actualmente lo que prevalece es el aumento de actitudes negativas, como temor, incomprensión, prejuicios, racismo y xenofobia ante las minorías procedentes de la inmigración. Un hecho constatable a medida que la presencia de dichas minorías se hace palpable en nuestra sociedad. Aunque poco a poco se va tomando conciencia de la relevancia social y política del fenómeno de la migración, y se adoptan medidas sociales de prevención de actitudes racistas, y de apoyo y acogida a la población migrante, queda aún un largo camino por recorrer para que la sociedad española viva un clima de efectiva integración del colectivo migrante (Pérez y Hernández Martín, 2003).

Es en el proceso de acercamiento a la interculturalidad, en donde la educación juega un papel relevante. Los procesos educativos formales y no formales son fundamentales a la hora de frenar el crecimiento de las ideas racistas y xenófobas, y apostar por el desarrollo de la tolerancia y el respeto a la diferencia.

En este sentido resulta importante conocer qué actitudes previas manifiestan, por ejemplo, los alumnos en el contexto escolar: prejuicios, estereotipos, intolerancia que, con frecuencia, se encuentran latentes; o, por el contrario, actitudes de respeto, igualdad y reacciones solidarias que pueden producirse y que influyen decisivamente en los comportamientos docentes y discentes cuando otros alumnos migrantes se incorporan a las aulas. Sólo a partir del estudio de estas actitudes, en contextos geográficos y sociales con una creciente población migrante, así como en otros en donde se empieza a producir el incremento de la misma, tímidamente, es posible elaborar y planificar propuestas de prevención e intervención que permitan eliminar o disminuir las frecuentes predisposiciones negativas hacia estos colectivos en la escuela.

Son las buenas prácticas educativas las que pueden favorecer un con- 
texto en el que la diferencia sea una fuente inagotable de posibilidades. El interés de la investigación educativa por prácticas de calidad o buenas prácticas docentes no es un tema novedoso. Este concepto está estrechamente vinculado a la cultura de calidad que debe caracterizar al sistema educativo y, por tanto, como señalan De Pablos y Jiménez (2007), el término de buena práctica educativa puede adquirir distintos usos y acepciones, entre las que se van a resaltar dos:

- Como una manera de ejemplificar una actividad realizada con resultados satisfactorios. El desarrollo de una buena práctica educativa responde a una visión compartida de "querer avanzar".

- Como reflejo/producto de la identidad de un contexto; se podría considerar como un buen instrumento para gestionar las diferencias y sacar a la luz lo singular y específico de ese contexto.

Por ello, una buena práctica educativa en el contexto escolar integra una experiencia educativa y una actividad docente que optimizan tanto los procesos como los resultados de aprendizaje. Y si se relaciona este concepto con el desarrollo de la interculturalidad, una buena práctica educativa para y desde la interculturalidad debe "proponer una mirada hacia la diversidad de los estudiantes, familias y comunidades que reconoce dicha diversidad y asume un compromiso por la igualdad de oportunidades y una escuela buena para todos" (Aguado, 2011, p.23).

\section{Marco empírico}

Como ya se ha avanzado, en este trabajo se pretende conocer y profundizar en las opiniones que los escolares tienen ante la progresiva llegada de migrantes que se ha generado en determinados entornos sociales y educativos. En concreto, el estudio se ha centrado en contextos educativos de Murcia y Salamanca, en los que la presencia de alumnado migrante en la primera es mucho más significativa y palpable que en la segunda. Se aspira a que los resultados obtenidos sirvan de pauta para diseñar, implementar y evaluar líneas de intervención que impulsen la educación para una ciudadanía más abierta y plural, en orden a promover una sociedad intercultural e inclusiva. 


\section{Diseño de investigación}

Se optó por un estudio de carácter comparativo con una primera aproximación descriptiva mediante la aplicación de un cuestionario, dentro de un diseño no experimental o ex-post-facto, al seleccionar grupos naturales, alumnos de centros educativos de carácter público de Murcia y de Salamanca de los niveles $5^{\circ}$ y $6^{\circ}$ de Primaria y $3^{\circ}$ y $4^{\circ}$ de Secundaria. Se analizaron las similitudes y diferencias entre una región que cuenta con una larga trayectoria vinculada a la presencia de alumnado migrante; y otra que recibe la incorporación de dichos escolares como un fenómeno relativamente reciente.

\section{Objetivos del estudio}

En consonancia con el diseño de la investigación los objetivos que se proponen son los detallados seguidamente:

- Analizar las opiniones de los escolares de Murcia y Salamanca respecto a la presencia de migrantes en el aula y otros contextos sociales, sus ideas y posibles prejuicios y estereotipos en cuanto a la interacción con ellos; y su percepción sobre la necesidad de formación en competencias interculturales en los centros escolares, para mejorar la interacción con personas de diversas culturas.

- Estudiar comparativamente las posibles diferencias en las opiniones y actitudes de los escolares, entre las dos regiones consideradas.

- Analizar la posible influencia de determinadas variables de carácter sociodemográfico sexo y nivel educativo- sobre dichas opiniones y actitudes.

- Apuntar algunas líneas de actuación educativa que contribuyan a fomentar la interacción entre alumnado migrante y autóctono para fomentar la convivencia positiva en el centro escolar.

\section{Instrumento}

El cuestionario constó de dos partes complementarias. La primera recogía información sobre variables de carácter sociodemográfico (sexo 
- nivel educativo - Primaria o Secundaria - y zona geográfica - Murcia y Salamanca-); y la segunda, variables directamente relacionadas con la opinión y actitudes acerca de la presencia de migrantes en contextos sociales y educativos cercanos: actitud hacia la migración en España, contacto con migrantes, actitudes de los compañeros de posibles migrantes, y opinión sobre la necesidad de formación en competencias interculturales para mejorar la interacción entre alumnado de diversas culturas.

La elaboración del cuestionario partió de la realización de una encuesta abierta, con objeto de recoger opiniones de estudiantes de Primaria y Secundaria sobre los temas señalados, en una serie de centros educativos de carácter público, a los que se tuvo acceso por su disponibilidad para participar. Las respuestas dadas por el alumnado a las distintas preguntas en esta encuesta inicial sirvieron de guía para la formulación del cuestionario, con respuestas cerradas en su mayor parte. Aunque para la elaboración de aquellas cuestiones relacionadas con la actitud, las razones por las que se produce la migración y la necesidad de una formación intercultural, se consideraron las principales respuestas abiertas que, con más frecuencia, habían sido dadas por los estudiantes en la encuesta inicial.

La primera versión del cuestionario fue sometida a juicio de expertos: un docente del área de Métodos de Investigación en la Universidad de Salamanca, dos profesores del área de Lengua y Literatura de dicha Universidad y cinco docentes de Educación Primaria y Secundaria. Las correcciones y sugerencias efectuadas por los mismos sirvieron para depurar cuestiones de forma y contenido, y elaborar el cuestionario definitivo. Se efectuó un pequeño estudio piloto en el que participaron estudiantes de Primaria y Secundaria de dos centros educativos, uno en Salamanca y otro en Murcia, con la finalidad de que el propio alumnado informase de posibles dudas a la hora de responder a las preguntas. Ello permitió la construcción del instrumento definitivo.

La aplicación se efectuó dentro de la jornada normal de clase, en grupos naturales. Se emplearon un total de diez sesiones de cuarenta y cinco minutos para dar algunas instrucciones y cumplimentar el cuestionario. Dado que la población objeto de estudio era menor de edad, se solicitó a los investigadores un certificado de delitos para poder acceder a los centros. Además, se pidió a los padres la firma de un escrito de autorización para que sus hijos pudiesen participar.

Se realizó una prueba de consistencia interna, el coeficiente Alfa de 
Crombach, para analizar en qué medida las distintas escalas medían el mismo constructo, lo que nos permitió confirmar la fiabilidad y las propiedades psicométricas del cuestionario. El coeficiente obtenido es de 0.81 , un resultado satisfactorio que indica que las preguntas formuladas a través de los ítems con respuestas cerradas, están bien correlacionadas y miden el constructo relacionado con las actitudes y opiniones hacia la llegada de estudiantes extranjeros a contextos escolares y sociales.

\section{Participantes}

Se definió la población objeto de estudio como el conjunto del alumnado de los colegios públicos de Murcia y de Salamanca de los cursos $5^{\circ}$ y $6^{\circ}$ de Primaria y $3^{\circ}$ y $4^{\circ}$ de la E.S.O. Concretamente, se trabajó con una muestra de 360 alumnos de 6 colegios públicos de ambas zonas, es decir 180 estudiantes procedentes de la Región de Murcia y 180 que residían en la Provincia de Salamanca. Exactamente la mitad de la muestra pertenecía a Educación Primaria y la otra mitad a Educación Secundaria. El $52.8 \%$ eran mujeres y el $47.2 \%$ varones en ambas localidades.

\section{Presentación y discusión de los resultados}

La presencia de migrantes en los centros educativos de Murcia es muy significativa en las aulas, donde se puede apreciar hasta veinte procedencias distintas del alumnado en el mismo centro. El $72.1 \%$ de los alumnos tenían cerca niños migrantes, tanto en el contexto escolar como social. Ello contrasta con lo que ocurre en los centros educativos de Salamanca, donde la presencia de migrantes resultó escasa en las aulas (1.7\%), frente al 3\% de la media nacional actual de alumnos extranjeros. Así mismo, el $58,3 \%$ de los alumnos tenían contacto con niños migrantes, bien en la clase (17\%), en el barrio (27.2\%), o en varios contextos a la vez (14.1\%).

\section{Actitud ante la llegada de migrantes a España}

Aunque no es elevado el porcentaje de alumnado de Salamanca (14\%), frente a un $6 \%$ de Murcia, que presenta una actitud de rechazo hacia la 
Ilegada de personas procedentes de otras culturas, sí resulta preocupante por lo que supone, teniendo en cuenta que la presencia de esta población en Salamanca no es tan significativa como en Murcia. Y los estudios de opinión reflejan constantemente un incremento cuantitativo de actitudes negativas, a medida que aumenta la incorporación de alumnado de otros países. Es previsible por tanto que, si no se ponen las medidas sociales y educativas oportunas, el porcentaje de estudiantes que manifiesta actitudes de rechazo continúe aumentando.

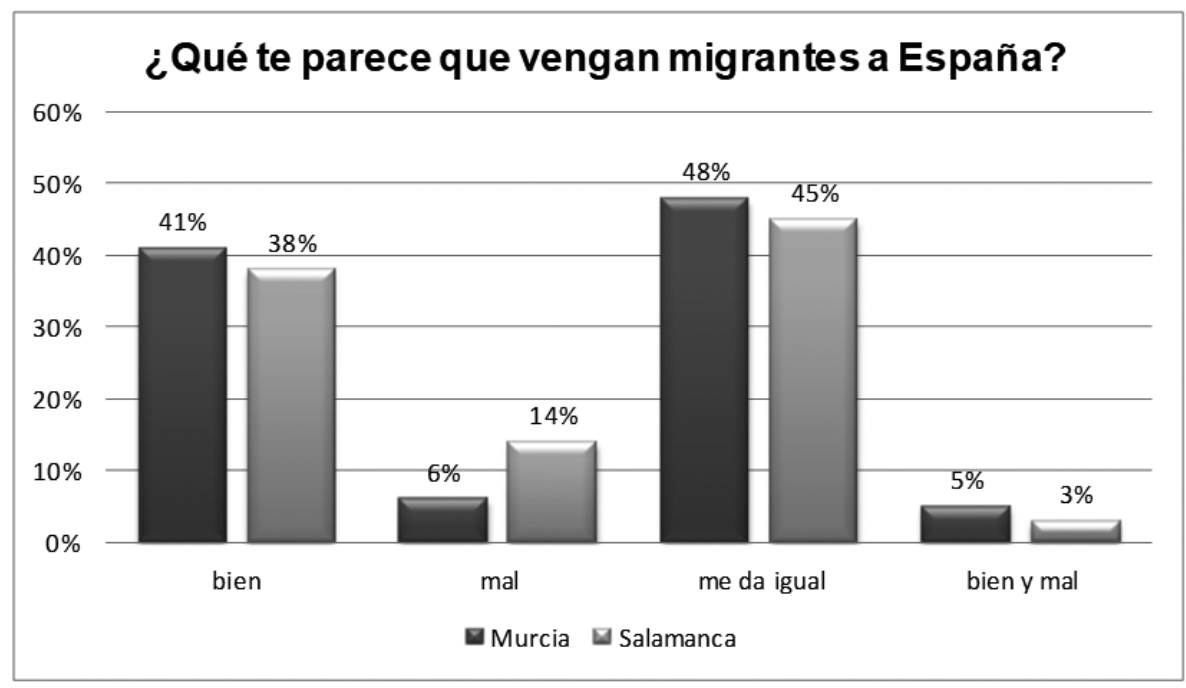

Gráfico 1. Actitudes hacia la llegada de migrantes a España.

Sin embargo, es curioso cómo a nivel descriptivo y en la muestra explorada se produce una cierta tendencia contraria a la que se ha apuntado y reflejan otros estudios (Retortillo y Rodríguez, 2008): es entre quienes tienen migrantes cerca, donde más personas aceptan la llegada de esta población $(45 \%)$. Sin la intención de generalizar, sí se puede apuntar tímidamente la idea de que cuando el número de migrantes es menor, como en Salamanca, tiende a ser más difícil aceptar las diferencias, debido, en cierto modo, al temor y miedo que genera lo desconocido. No obstante, cuando el número de migrantes aumenta, como en el caso de Murcia, el intercambio y la interacción compartida entre diferentes culturas se produce de manera natural. Así, mientras Salamanca se encuentra en una fase intermedia de recepción de migrantes, Murcia, sin embargo, está inmersa en soslayar otras dimensiones de los proyectos 
migratorios. A pesar de lo apuntado desde una perspectiva descriptiva, no se han observado diferencias en lo que respecta a las actitudes ante la llegada de migrantes entre las dos zonas geográficas analizadas $\chi^{2}(2$, $\mathrm{N}=360)=1.72, \mathrm{p}=0.59$ ).

En relación a la influencia de las variables sociodemográficas consideradas en las actitudes de los escolares hacia la llegada de migrantes a España, los análisis realizados confirman que el sexo no es una variable que influya en dichas actitudes $\left(\chi^{2}(2, N=360)=1.04, p=0.56\right)$. Sí se encontraron diferencias en lo que respecta al nivel educativo, de modo que son los alumnos de Educación Primaria quienes manifiestan actitudes más positivas hacia la llegada de personas procedentes de otros países $\left(\chi^{2}(2, N=180)=10.35, p=0.005\right)$.

\section{Razones de la actitud ante la llegada de migrantes}

Estas razones se recogen en la tabla 1. Se pueden apreciar los argumentos más frecuentes entre los alumnos con actitud positiva; un 31,9\% en Murcia refiere "La necesidad que tienen los migrantes de oportunidades y ayudas por tener problemas en su país" y un 29.4\% en Salamanca que precisa lo mismo. El 28,1\% en Murcia señala que "todos tienen derecho a ir y vivir donde quieran", frente un $27,9 \%$ en Salamanca. La posibilidad de "poder conocer otras culturas, costumbres" es destacada con porcentajes similares por ambas muestras. Si se observan los indicadores más significativos sobre el alumnado con una actitud negativa, resaltan los prejuicios y estereotipos basados en datos subjetivos y no realistas, tales como que "nos quitan puestos de trabajo u otro tipo de bienes a los españoles". Un $11 \%$ de los estudiantes de Murcia y un $15 \%$ de los de Salamanca argumentan también que "nos invaden". Indicadores que los escolares interiorizan de forma acrítica en contextos cercanos como familiar, escolar y a través de los medios de comunicación.

No existen diferencias entre ambas localidades en cuanto a la actitud que manifiestan ante la llegada de migrantes $\left(\chi^{2}(2, \mathrm{~N}=360)=1.35, \mathrm{p}=\right.$ 0.67). Tampoco se dan estas diferencias atendiendo al nivel educativo $\left(\chi^{2}(2, N=360)=1.25, \mathrm{p}=0.56\right)$ y al $\operatorname{sexo}\left(\chi^{2}(2, \mathrm{~N}=360)=1.29, \mathrm{p}=\right.$ $0.89)$. 
Actitudes de los escolares ante la llegada de población migrante: Estudio comparativo y aproximación al desarrollo de buenas prácticas educativas Mohamed Chamseddine Habib Allah y Azucena Hernández Martín

Tabla 1

Actitudes hacia la llegada de migrantes por parte de los escolares (Elaboración propia)

\begin{tabular}{|c|c|c|c|c|}
\hline \multirow{2}{*}{ Actitud hacia la llegada de migrantes } & \multicolumn{2}{|c|}{ Murcia } & \multicolumn{2}{|c|}{ Salamanca } \\
\hline & Bien & Mal & Bien & Mal \\
\hline $\begin{array}{l}\text { Tienen problemas en su país y necesitan opor- } \\
\text { tunidad y ayuda }\end{array}$ & $31.9 \%$ & & $29.4 \%$ & \\
\hline $\begin{array}{l}\text { Todos tienen derecho a vivir bien e ir donde } \\
\text { quieran }\end{array}$ & $28.1 \%$ & & $27.9 \%$ & \\
\hline Mantienen sus costumbres, cultura, idioma, etc. & $24.6 \%$ & & $23.5 \%$ & \\
\hline Somos más habitantes en España & $7.5 \%$ & & $11.8 \%$ & \\
\hline Nos quitan el trabajo y/u otros bienes & & $39 \%$ & & $56 \%$ \\
\hline Nos invaden & & $11 \%$ & & $16 \%$ \\
\hline Es mejor que cada uno se quede en su país & & $7 \%$ & & $4 \%$ \\
\hline \multicolumn{5}{|l|}{ Mejor si vienen pocos, mal si vienen muchos } \\
\hline Bien si necesitan trabajo, mal si delinquen & & $23 \%$ & & \\
\hline \multicolumn{5}{|l|}{$\begin{array}{l}\text { Bien porque hacen trabajos que nadie quiere, } \\
\text { mal si no tienen papeles }\end{array}$} \\
\hline Otros & $4.8 \%$ & $9 \%$ & $5.9 \%$ & $8 \%$ \\
\hline NS/NC & $3.1 \%$ & $11 \%$ & $1.5 \%$ & $16 \%$ \\
\hline $\operatorname{TOTAL}(n=360)$ & $100 \%$ & $100 \%$ & $100.0 \%$ & $100 \%$ \\
\hline
\end{tabular}

\section{Migrantes cerca}

Quienes han positivamente, razonan su respuesta señalando que eso "le permite o permitiría conocer otras cultura, otras costumbres". Con menor relevancia aducen que "posibilitaría hacer nuevos amigos" o "que los migrantes le resultan buenos y/o agradables". El 42.9\% de los que han respondido que no les gustaría, no saben justificar su respuesta. Algunos aportan razones dispersas y arbitrarias como que: "No hablan bien", "no se les entiende", "no los conozco", "no me gustan", "este país es sólo para españoles", "son peligrosos", etc. 


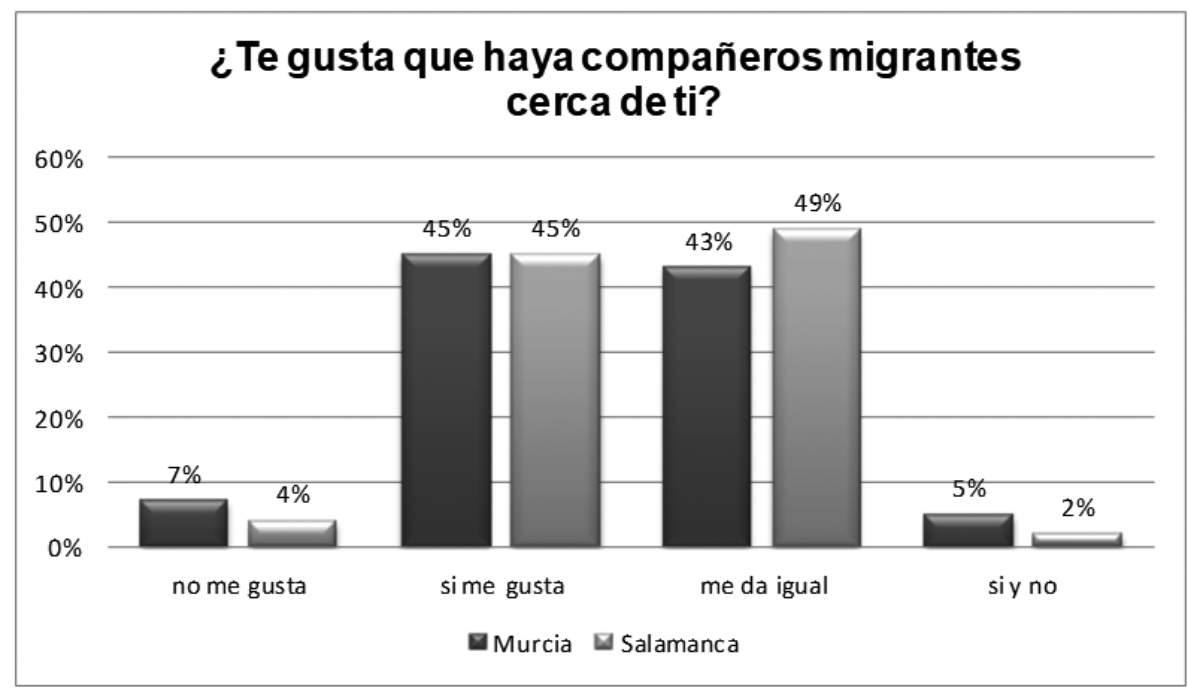

Gráfico 2. Agrado por la cercanía de compañeros migrantes.

Las estudiantes manifiestan en mayor medida que el colectivo varón su agrado por la cercanía de migrantes $\left(\chi^{2}(2, \mathrm{~N}=190)=9.15, \mathrm{p}=0.0051\right)$. No hay diferencias atendiendo a la zona de procedencia $\left(\chi^{2}(2, N=\right.$ $360)=1.05, \mathrm{p}=0.76)$, $\mathrm{ni}$ al nivel educativo $\left(\chi^{2}(2, \mathrm{~N}=360)=2.35, \mathrm{p}=\right.$ $0.61)$.

\section{Influencia de la presencia de migrantes en la clase}

Entre quienes responden en ambas localidades que dicha presencia no influiría razonan que como todos son iguales, no tiene por qué afectar ni en sentido positivo ni negativo. Sólo unos pocos, de los que piensan positivamente, argumentan abiertamente una postura de máxima interculturalidad, señalando que las diferencias son enriquecedoras (Gráfico 2). 


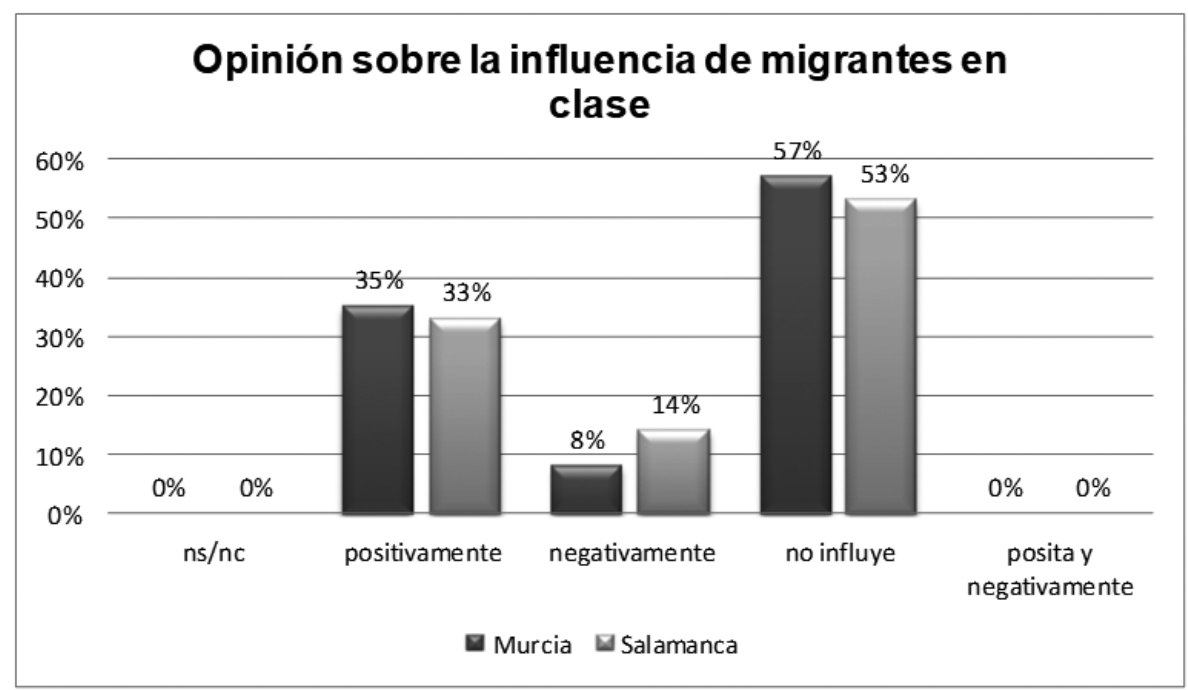

Gráfico 3. Opinión sobre la influencia de migrantes en clase.

En la Tabla 2 se aprecian las razones dadas por quienes consideran que la presencia de migrantes en clase influiría positiva o negativamente. Más de la mitad de los que piensan que influiría en sentido positivo, creen que de esta forma "se conocerían más sus costumbres". Otras respuestas son: "todos somos iguales". O bien, "se harían nuevos amigos y amistades". Los que consideran que la presencia de colectivos migrantes influye negativamente, señalan que"serían discriminados en clase".

La zona geográfica no es una variable que tenga importancia a la hora de considerar la influencia en clase de estos colectivos $\left(\chi^{2}(2, N=360)\right.$ $=1.31, \mathrm{p}=0.61)$. El nivel educativo tampoco $\left(\chi^{2}(2, \mathrm{~N}=360)=1.90, \mathrm{p}=\right.$ $0.62)$.

Las estudiantes presentan actitudes más positivas al señalar que el tener a estudiantes procedentes de otros países en clase permitiría conocer otras culturas, otras costumbres, y realizar intercambios culturales $\left({ }^{2}(2\right.$, $\mathrm{N}=190)=9.80, \mathrm{p}=0.0052$ ). 
Actitudes de los escolares ante la llegada de población migrante: Estudio comparativo y aproximación al desarrollo de buenas prácticas educativas Mohamed Chamseddine Habib Allah y Azucena Hernández Martín

Tabla 2

Actitudes hacia la llegada de migrantes por parte de los escolares (Elaboración propia

\begin{tabular}{|c|c|c|c|c|}
\hline \multirow{2}{*}{$\begin{array}{l}\text { De qué forma considera } \\
\text { que influyen los migran- } \\
\text { tes en la clase }\end{array}$} & \multicolumn{2}{|c|}{ Murcia } & \multicolumn{2}{|c|}{ Salamanca } \\
\hline & Positivamente & $\begin{array}{l}\text { Negati- } \\
\text { vamente }\end{array}$ & Positivamente & $\begin{array}{l}\text { Negati- } \\
\text { vamente }\end{array}$ \\
\hline $\begin{array}{l}\text { Se conocen otras cultura, } \\
\text { costumbres, etc. }\end{array}$ & $60.3 \%$ & & $57.9 \%$ & \\
\hline $\begin{array}{l}\text { Bien ya que todos somos } \\
\text { iguales }\end{array}$ & $22.8 \%$ & & $24.6 \%$ & \\
\hline $\begin{array}{l}\text { Haremos amigos y nue- } \\
\text { vas amistades }\end{array}$ & $16.9 \%$ & & $17.5 \%$ & \\
\hline $\begin{array}{l}\text { Todos los compañeros } \\
\text { los discriminarían }\end{array}$ & & $39.8 \%$ & & $47.6 \%$ \\
\hline Son peligrosos & & $15.9 \%$ & & $17.8 \%$ \\
\hline Otros & & $22.8 \%$ & & $23.7 \%$ \\
\hline $\mathrm{NS} / \mathrm{NC}$ & & $21.5 \%$ & & $10.9 \%$ \\
\hline TOTAL & $100 \%$ & $100 \%$ & $100 \%$ & $100 \%$ \\
\hline
\end{tabular}

\section{Relaciones con migrantes}

Son los escolares de la región de Murcia quienes tienen menos dificultades para relacionarse en igualdad de condiciones (Gráfico 4), tanto con estudiantes autóctonos, como con aquellos de otros orígenes $\left(\chi^{2}(2, N=\right.$ 180) $=9.90, \mathrm{p}=0.004)$.

Los análisis realizados confirman que el sexo es nuevamente una variable que influye a la hora de considerar diferencias de relación con estudiantes de otras culturas. Las chicas estiman en mayor grado que dichas relaciones no tienen por qué ser diferentes $\left(\chi^{2}(2, N=190)=10.21, p\right.$ $=0.005)$. También el nivel educativo marca dichas diferencias, de modo que los estudiantes de Educación Primaria tienden a pensar que estas relaciones son iguales que las que pueden mantener con compañeros autóctonos $\left(\chi^{2}(2, N=180)=9.60, p=0.0046\right)$. 


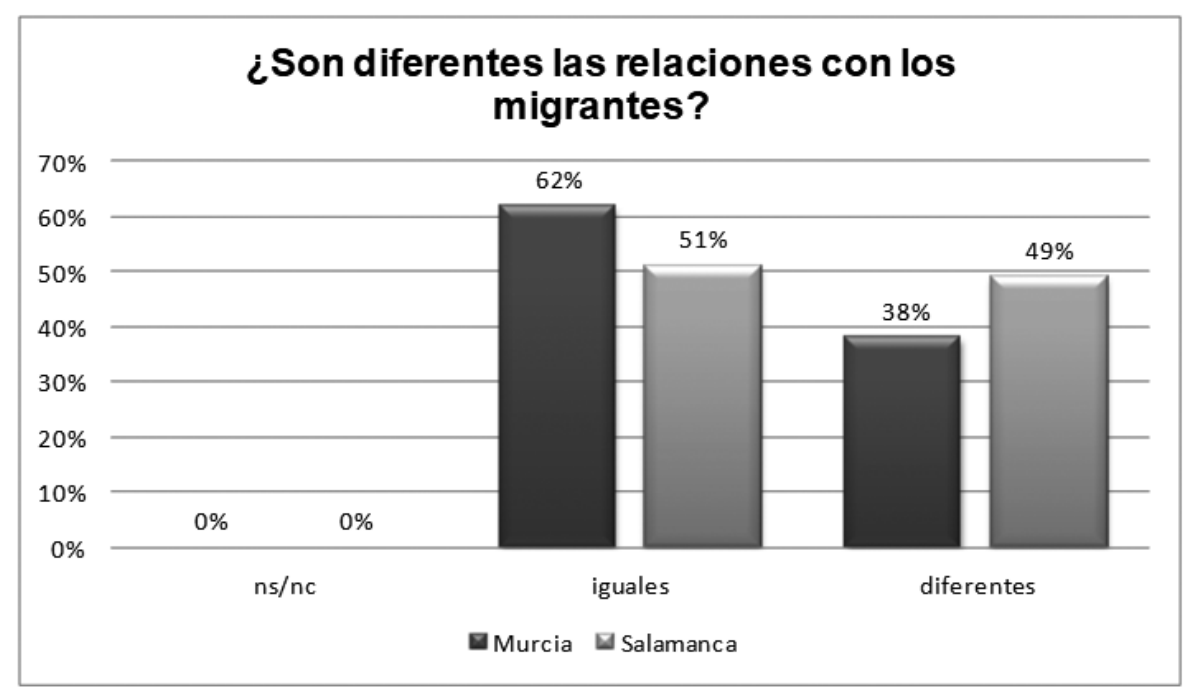

Gráfico 4. Diferencias en las relaciones con población migrante.

\section{Trato a los migrantes}

Las estudiantes son más sensibles hacia el comportamiento inadecuado que reciben en ocasiones estos escolares $\left(\chi^{2}(2, N=190)=9.90, p\right.$ $=0.0049)$. No se han encontrado diferencias significativas en lo que respecta a esta variable, atendiendo al nivel educativo $\left(\chi^{2}(2, N=360)\right.$ $=1.44, \mathrm{p}=0.78)$ y a la zona de procedencia $\left(\chi^{2}(2, \mathrm{~N}=190)=1.11, \mathrm{p}=\right.$ $0.710)$.

La principal razón que señalan quienes opinan que no se les trata bien es que "existen prejuicios, discriminación e intolerancia" (81.6\%); el $5.9 \%$ cree que "suscitan miedo o peligro". La mayoría de los que estiman que no se les trata ni bien ni mal no razonan su respuesta, aunque algunos manifiestan que "hay personas que les tratan bien y otros mal". El $17.6 \%$ habla de un aprendizaje mutuo, y el $29.5 \%$ ofrecen respuestas diversas, no encuadrables en ninguna categoría. 


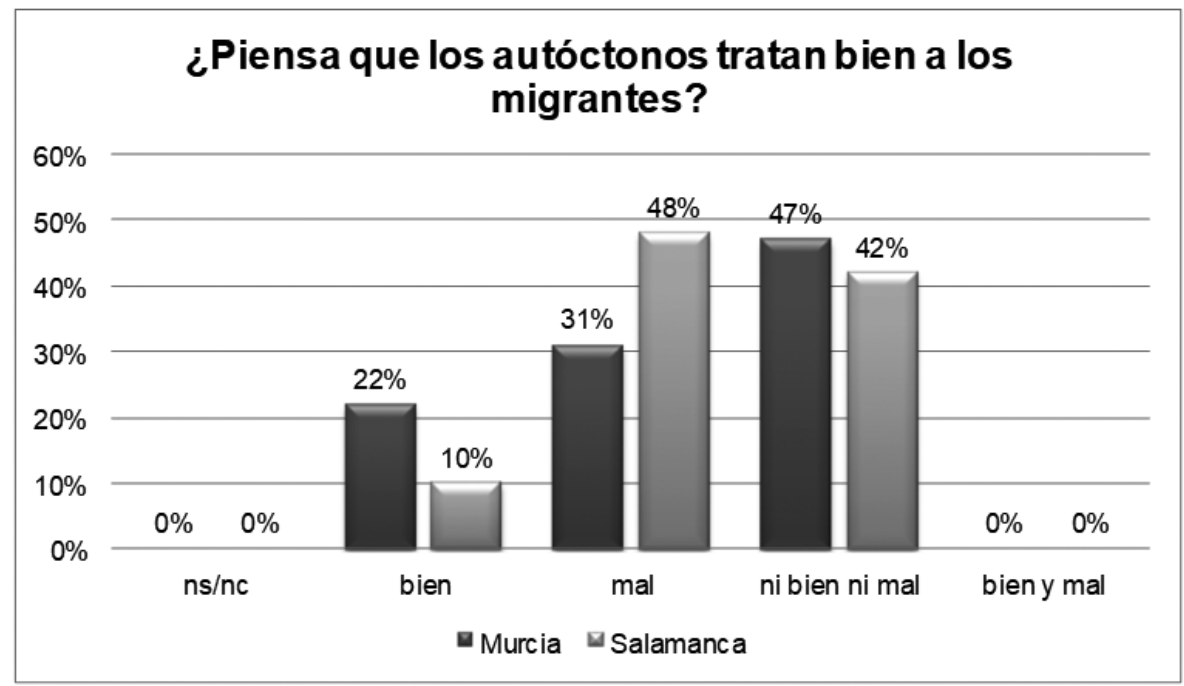

Gráfico 5. Trato que recibe el colectivo migrante por parte de la población autóctona.

\section{Necesidad de formación en competencias interculturales en el centro educativo}

Tal como se aprecia en la Tabla 3, el $43.2 \%$ de los escolares murcianos, frente al $38.4 \%$ de los estudiantes salmantinos, manifiesta que es necesaria una formación en competencias interculturales "para mejorar las relaciones y evitar prejuicios". Algunos consideran que adquirir dichas competencias permitiría "transmitir que todos somos iguales" y "conocer mejor su cultura, lengua y costumbres".

En relación al alumnado que no es consciente de la necesidad de formación, indican que "no hace falta porque somos iguales", o que "no hace falta porque casi no hay migrantes", aspecto que indican fundamentalmente los escolares de Salamanca. Se identifican, por tanto, diferencias en lo que se refiere a la formación en competencias interculturales, considerando las dos localidades $\left({ }^{2}(2, N=190)=10,36, p=\right.$ 0.0053). Es en Salamanca donde no perciben tanto como en Murcia la necesidad de formación, debido probablemente a la menor presencia de alumnado migrante en las aulas. El sexo y el nivel educativo no son variables reseñables por lo que respecta a esta variable. 
Actitudes de los escolares ante la llegada de población migrante: Estudio comparativo y aproximación al desarrollo de buenas prácticas educativas Mohamed Chamseddine Habib Allah y Azucena Hernández Martín

Tabla 3

Necesidad de formación en competencias interculturales en el colegio (Elaboración propia).

\begin{tabular}{|c|c|c|c|c|}
\hline \multirow{2}{*}{$\begin{array}{l}\text { Piensas que es necesaria la formación en } \\
\text { competencias interculturales en el colegio }\end{array}$} & \multicolumn{2}{|c|}{ Murcia } & \multicolumn{2}{|c|}{ Salamanca } \\
\hline & $\mathrm{Si}$ & No & $\mathrm{Si}$ & No \\
\hline \multicolumn{5}{|l|}{ Razones } \\
\hline $\begin{array}{c}\text { Para mejorar las relaciones y evitar prejui- } \\
\text { cios }\end{array}$ & $43.2 \%$ & & $38,4 \%$ & \\
\hline Para transmitir que todos somos iguales & $17.3 \%$ & & $15,1 \%$ & \\
\hline $\begin{array}{l}\text { Para conocer mejor su cultura, lengua y } \\
\text { costumbres }\end{array}$ & $20.1 \%$ & & $14,0 \%$ & \\
\hline $\begin{array}{l}\text { Hay que formarse en competencias inter- } \\
\text { culturales }\end{array}$ & $9.2 \%$ & & $8,1 \%$ & \\
\hline Van a venir muchos migrantes & $2.1 \%$ & & $7,0 \%$ & \\
\hline No hace falta porque somos iguales & & $39.9 \%$ & & $41,2 \%$ \\
\hline $\begin{array}{c}\text { No hace falta porque casi no hay migran- } \\
\text { tes }\end{array}$ & & $11.8 \%$ & & $17,6 \%$ \\
\hline Porque no quiero que vengan & & $11.1 \%$ & & $11,8 \%$ \\
\hline Otras & 7.0 & $14.3 \%$ & $10,4 \%$ & $5,9 \%$ \\
\hline $\mathrm{NS} / \mathrm{NC}$ & 1.1 & $22.9 \%$ & $7,0 \%$ & $23,5 \%$ \\
\hline TOTAL & $100 \%$ & $100 \%$ & $100 \%$ & $100 \%$ \\
\hline
\end{tabular}

\section{Conclusiones}

Los resultados obtenidos permiten la consecución de los objetivos previstos en el estudio. Como se ha señalado a lo largo del presente artículo, Murcia cuenta con una larga trayectoria en relación a la presencia de alumnado migrante procedente de múltiples países. Por su parte, en Salamanca dicha presencia, tanto a nivel social como escolar, es un fenómeno más bien reciente. Sin embargo, en comparación con la localidad murciana, se observa en algunas de las respuestas, que las actitudes de los estudiantes de Salamanca son de cierta reticencia hacia la presencia de compañeros migrantes; lo que se puede traducir en algunos casos en prejuicios y rechazos basados en argumentos poco ajustados a la realidad. Este estudio evidencia que el desconocimiento y un contacto todavía escaso con población migrante en los contextos escolares puede generar cierto grado de desconfianza hacia aquello que se desconoce, 
en lugar de una interacción más natural, fruto de una trayectoria amplia de convivencia intergrupal.

También es destacable la percepción generalizada del trato inadecuado que recibe el colectivo migrante, y especialmente las estudiantes de la muestra. También son ellas las que muestran una mayor sensibilidad hacia la cercanía de migrantes y estiman que las relaciones con ellos no tienen por qué ser diferentes. Poseen más interés por conocer sus peculiaridades culturales, además de manifestarse más a favor de que los contextos educativos deberían formar al alumnado en competencias interculturales para mejorar la convivencia escolar y social. El sexo no es una variable que influya en las actitudes ante la llegada de migrantes entre las dos zonas geográficas analizadas.

La zona geográfica no es una variable determinante a la hora de considerar la influencia en clase de migrantes, como tampoco lo ha sido el nivel educativo. Sin embargo, sí se consideran posibles diferencias en las relaciones con población migrante; son los escolares de la región de Murcia quienes tienen menos dificultades para establecer esta relación.

También es diferente la forma de percibir la necesidad de formación en competencias interculturales, si se consideran ambas localidades. Los estudiantes de Salamanca perciben menos la necesidad de esta formación, aspecto que se puede justificar si se considera que hay una menor presencia de colectivo migrante en las aulas.

Las actitudes positivas son más frecuentes entre los escolares de Educación Primaria, quienes tienden a pensar, además, que las relaciones con estos compañeros son iguales que las que pueden desarrollar con cualquier otro. Ello puede deberse a la mayor atención que se está prestando en los contextos educativos, a una educación intercultural intencional que cale en el sentir y en el hacer diario.

El último objetivo que se planteaba iba orientado al establecimiento de algunas líneas de actuación educativa que contribuyesen a fomentar la convivencia positiva en el centro escolar, a través de una adecuada interacción entre alumnado migrante y autóctono.

Se ha de tener en cuenta, en primer lugar, que la incorporación del alumnado migrante en el contexto escolar presenta nuevos retos e implicaciones que conducen a cambios a nivel organizativo, metodológico y curricular, y que requieren el diseño, implementación y evaluación de nuevas estrategias educativas, o de otras prácticas, desde una perspectiva inclusiva e intercultural. 
Esto supone tener en cuenta dos reflexiones teóricas, antes de plantear algunas líneas de actuación en este sentido. La primera está vinculada con una pedagogía intercultural que facilite una mayor comprensión, apertura y enriquecimiento mutuo de los alumnos de diferentes culturas. Y la segunda se relaciona con la pedagogía de la diversidad, que debería ser un principio de cada acto educativo para aumentar la creatividad cultural de cada alumno, la sensibilidad y el conocimiento de otros comportamientos o interpretaciones de la realidad (Schmidtke, 2007).

La primera línea de actuación se orienta a la necesidad de abordar la formación específica del profesorado en atención a alumnado migrante, así como la adquisición de competencias comunicativas e interculturales, implementando actuaciones y estrategias inclusivas como un derecho y no como respuesta académica a las nuevas necesidades del alumnado migrante (Escudero, 2012). Esta formación docente es clave para responder a las necesidades y los retos que se plantean en contextos sociales cada vez más multiculturales. Pero, es aún más necesaria en un momento muy delicado en lo que respecta a actitudes de rechazo generalizado hacia ciertos colectivos migrantes, como consecuencia de actos terroristas acaecidos en distintas ciudades del mundo. Ello ha generado, en una parte significativa de la población, actitudes xenófobas y de rechazo frontal hacia todo aquello que tenga que ver con lo "externo y desconocido".

El papel del profesor y su formación, así como la que deben adquirir los futuros docentes en los planes de formación inicial, cobra una especial relevancia, pues son o serán las personas encargadas de desarrollar en los alumnos distintas destrezas interculturales en un entorno cada vez más heterogéneo, y de enseñarles a comprender perspectivas y valores diferentes a los propios.

En definitiva, los docentes han de ser capaces de transformar la mirada de los jóvenes como principio para valorar la diferencia (Hernández Martín e Iglesias, 2017). Es necesario, por tanto, gestionar la formación y desarrollo profesional docente desde una escuela integral que proporcione conocimientos, procedimientos y actitudes para la adquisición de competencias interculturales. Una escuela participativa que desarrolle actuaciones basadas en la reflexión crítica y la toma de decisiones consensuada. Una escuela inclusiva, que garantice la igualdad de condiciones y la calidad educativa de todo el alumnado. Una escuela intercultural que impulse espacios de conocimiento y valoración de la diversidad 
cultural. Y, por último, una escuela que actúe en red, implicando a todos los actores de la comunidad educativa (Chamseddine, 2018).

Otra línea de actuación tiene que ver con el desarrollo de competencias interculturales en los estudiantes, entendidas como conjunto de habilidades basadas en el intercambio comunicativo con personas pertenecientes a otros entornos socioculturales, donde intervienen varios elementos como destrezas de interpretación, habilidades de descubrimiento e interacción, concienciación cultural crítica, curiosidad y apertura hacia los demás. Y todo ello con la implicación de tres posibles fuentes como son la familia, primera institución educativa, la didáctica que proporcionan los libros de texto y otros materiales impresos, y la mediática que facilitan los medios de comunicación de masas (Sánchez, 2016). Desarrollar competencias interculturales supone, en consecuencia, la existencia de recursos variados en las aulas y el acceso a fuentes de información y estudio de diversa índole. Pero, además, implica la apuesta por el desarrollo de comunidades de aprendizaje, entendidas como espacios educativos en donde todas las instancias cercanas a los estudiantes (familia, escuela, comunidad), desempeñan un papel importante en la definición y desarrollo de buenas prácticas educativas interculturales.

Dichas prácticas, tercera línea de actuación, se caracterizan por enseñar de otro modo a todos los estudiantes y no a algunos definidos desde un principio como diferentes (Aguado Odina, 2005; 2011).Y parten de la revisión y reflexión sobre las prácticas habituales en el centro y en las aulas; y sobre si lo que se hace es aquello que realmente se quiere hacer para, a partir de ahí, establecer actuaciones y modos de pensar relacionados con tres cuestiones básicas: "cómo se entiende la diversidad cultural; qué debería hacer el centro escolar como tal; qué pueden hacer los profesores en relación con la enseñanza y la evaluación" (Aguado Odina, 2011, p. 38).

En esta línea, las prácticas educativas interculturales, para que sean sistemáticas y consistentes en el tiempo, deben superar la visión restrictiva y anecdótica que tiende a relacionar la diversidad con el conflicto, la migración, la pobreza o las necesidades educativas; y constriñe dichas prácticas a días especiales dedicados a la interculturalidad en los centros y/o a las actividades que sólo se desarrollan en los momentos de tutoría. Todo ello, si bien contribuye a desarrollar una sensibilidad momentánea, no necesariamente genera cambios de actitud consistentes en el tiempo. La transversalidad y la intencionalidad en el tratamiento de este tema es muy relevante, así como la diversidad de procedimientos que los docen- 
tes utilicen para ello: proyectos, lecturas, debates, asambleas, dramatizaciones, video-forum, son algunas de las posibles actividades para presentar información, analizar prejuicios y estereotipos y clarificar valores.

No se pueden olvidar, por último, las potencialidades de las TIC, y concretamente de Internet, para permitir que tanto docentes como estudiantes adquieran una visión más amplia de otra cultura, y despierten su curiosidad e interés por el entendimiento cultural, a través de un proceso de inmersión digital en la misma (Hernández Martín e Iglesias, 2017). Priegue y Leiva (2012) exponen cuatro aportaciones básicas de las Tecnologías al contexto intercultural. Dichas aportaciones son especialmente interesantes porque permiten vislumbrar el poder de las herramientas tecnológicas como un entorno ecológico específico desde el que trabajar la interculturalidad (Priegue y Leiva, 2012, p. 3):

\begin{abstract}
"Motivar a los más jóvenes a participar en la generación de comunidades virtuales. Promover el conocimiento y reconocimiento de las diferencias culturales como claves positivas de enriquecimiento personal y social. Facilitar la comunicación intercultural a través del contacto de personas y culturas muy diversas. Favorecer la participación activa y colaborativa gracias al empleo sistemático de herramientas virtuales de la Web 2.0"
\end{abstract}

En esta línea de consideración de la TIC se considera interesante remitir al lector a otro trabajo previo en el que se propone un banco de recursos virtuales que sirva al profesorado, a los estudiantes, y a otros profesionales de la educación, para trabajar la atención a la diversidad y la interculturalidad, desde entornos propiamente tecnológicos (Hernández Martín e Iglesias, 2017).

\title{
Referencias
}

Aguado, T (2005). La educación intercultural en la práctica escolar. Investigación en el ámbito español. XXI, Revista de Educación, 7, 43-51.

Aguado, T. (2011). El enfoque intercultural en la búsqueda de buenas prácticas escolares. Revista Latinoamericana de Inclusión Educativa, 5(2), 23-42.

Carrasco, M.J. y Coronel, J.M. (2017). Percepciones del profesorado sobre la gestión de la diversidad cultural: Un estudio cualitativo. Educación XX1, 20(1), 75-98.

Cea D'ancona, M.A. (2011). La compleja detección del racismo y la xenofobia a través de encuesta. Un paso adelante en su medición. Revista Española de Investigaciones Sociológicas (Reis), 125, 13-45. 
Actitudes de los escolares ante la llegada de población migrante: Estudio comparativo y aproximación al desarrollo de buenas prácticas educativas Mohamed Chamseddine Habib Allah y Azucena Hernández Martín

Checa, J.C. y Arcona, A. (2011). Españoles ante la inmigración: el papel de los medios de comunicación. Comunicar, 36(19), 141-149.

Chamseddine, M. (2018). Polarización escolar en España. Retos e implicaciones. International Journal of Educational Research and Innovation (IJERI), 9, 189-205.

De Juan Vigaray, M; Parra, M.C. y Beltrán, M.A. (2014). Multiculturalidad, interculturalidad y desarrollo personal en el EEES. Revista de Comunicación de la SEECI, Año XVII, 35, 46-63.

De Pablos, J. y Jiménez, R. (2007). Buenas prácticas con TIC apoyadas en las Políticas Educativas: claves conceptuales y derivaciones para la formación en competencias ECTS. Revista Latinoamericana de Tecnología Educativa, 6 (2), 15-28.

Escudero, J.M. (2012). La educación inclusiva, una cuestión de derecho. Revista Educatio Siglo XXI, 30 (2),109-128.

Hernández, A. e Iglesias, A. (2017). La importancia del desarrollo de competencias digitales e informacionales para el desarrollo de una competencia intercultural. Interacçoes, 13(43), 205-232.

MECD (2016). Informe estadístico 2016. Madrid: Servicio de Publicaciones del Ministerio de Educación, Cultura y Deportes.

Retortillo, Á. y Rodríguez, H. (2008). Actitudes de aculturación y prejuicio étnico en los distintos niveles educativos: un enfoque comparado. REIFOP, 11(2), 61-71.

Richardson, T. (2011). Interrogating the Trope of the Door in Multicultural Education: Framing Diplomatic Relations to Indigenous Political and Legal Difference. Educational Theory, 61(3), 295-310.

Pérez, M.D. y Hernández, A. (2003). Actitudes de los escolares ante la llegada de inmigrantes en contextos sociales y educativos de Salamanca. En P.A. Luque, L.V. Amador y Malagón, J.L. Educación Social e Inmigración (pp. 128-138). Sevilla: Diputación de Sevilla.

Pérez Serrano, G. y Pérez De Guzmán, M.V. (2011). Aprender a convivir. Madrid: Narcea.

Santos, M.A. y Lorenzo, M. (2012). Estudios de Pedagogía Intercultural. Barcelona: Octaedro.

Sánchez, G. (2016). La comunicación, interacción y entendimiento en la Europa multicultural a través de la actualización de los programas de formación del profesorado especialista en lengua extranjera. Revista Educatio Siglo XXI, 34 (3), 181-198.

Schmidtke, H.P. (2007). El fenómeno de la inmigración y su respuesta pedagógico-intercultural en Alemania: Hacia la pedagogía de la diversidad. En J.L. Álvarez Castillo y L. Batanaz Palomares (Eds.). Educación intercultural e inmigración: de la teoría a la práctica, (pp. 77-92). Madrid: Editorial Biblioteca Nueva, S.L.

\section{Notas}

El artículo está vinculado al proyecto REF.: I+D+I EDU2015-68718REDT (Ministry of Economic Affairs and Competitiveness). Programa Estatal de Fomento de la Investigación Científica y Técnica de Excelencia, 
Actitudes de los escolares ante la llegada de población migrante: Estudio comparativo y aproximación al desarrollo de buenas prácticas educativas Mohamed Chamseddine Habib Allah y Azucena Hernández Martín

Subprograma Estatal de Generación de Conocimiento, en el marco del Plan Estatal de Investigación Científica y Técnica y de Innovación 20132016 